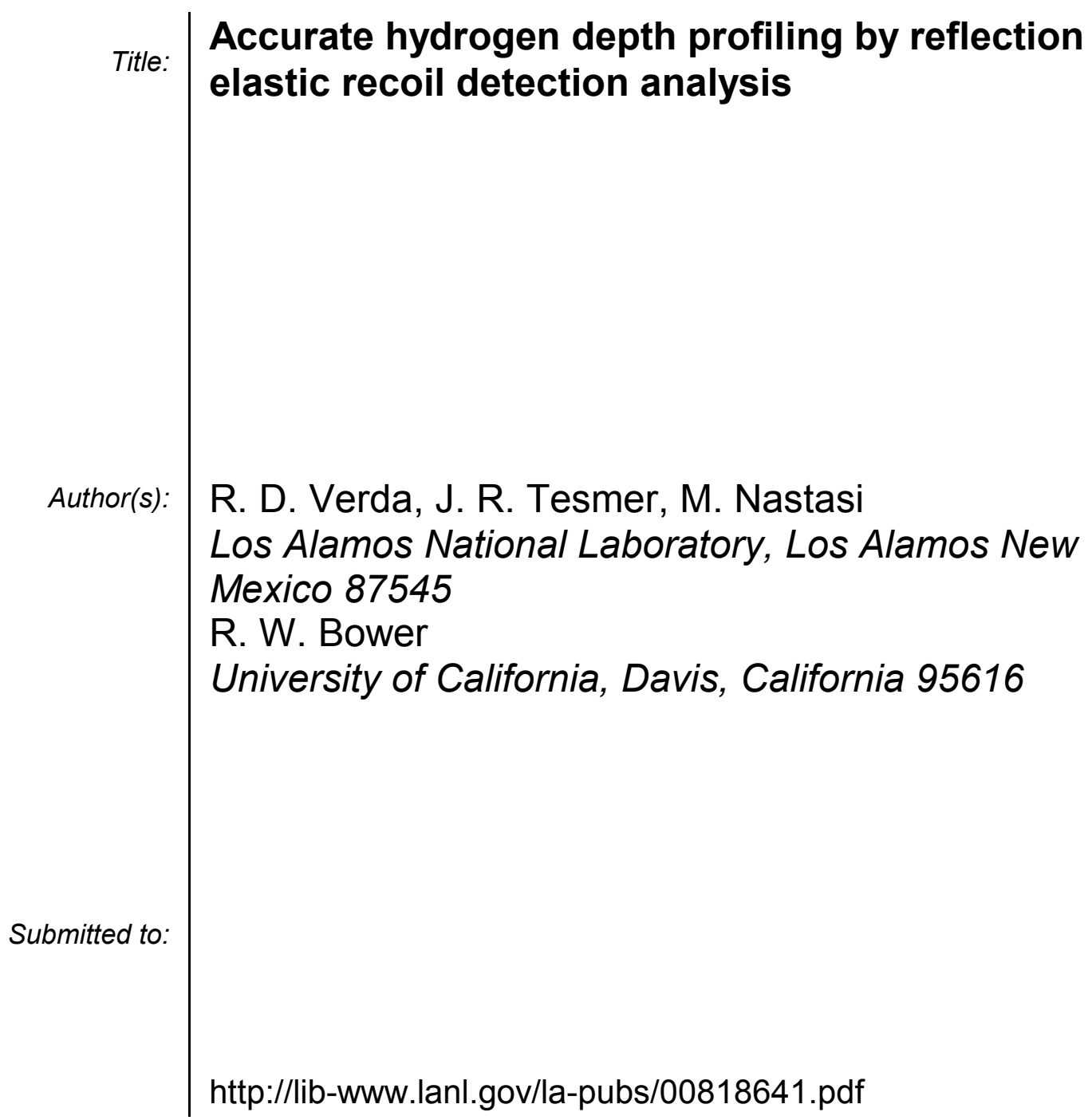

Los Alamos National Laboratory, an affirmative action/equal opportunity employer, is operated by the University of California for the U.S. Department of Energy under contract W-7405-ENG-36. By acceptance of this article, the publisher recognizes that the U.S. Government retains a nonexclusive, royaltyfree license to publish or reproduce the published form of this contribution, or to allow others to do so, for U.S. Government purposes. Los Alamos National Laboratory requests that the publisher identify this article as work performed under the auspices of the U.S. Department of Energy. Los Alamos National Laboratory strongly supports academic freedom and a researcher's right to publish; as an institution, however, the Laboratory does not endorse the viewpoint of a publication or guarantee its technical correctness. 


\title{
Accurate hydrogen depth profiling by reflection elastic recoil detection analysis
}

R. D. Verda, J. R. Tesmer, M. Nastasi

Los Alamos National Laboratory, Los Alamos New Mexico 87545

R. W. Bower

University of California, Davis, California 95616

\begin{abstract}
A technique to convert reflection elastic recoil detection analysis spectra to depth profiles, the channel-depth conversion, was introduced by Verda, et al $\left[^{1}\right]$. But the channel-depth conversion does not correct for energy spread, the unwanted broadening in the energy of the spectra, which can lead to errors in depth profiling. A work in progress introduces a technique that corrects for energy spread in elastic recoil detection analysis spectra, the energy spread correction $\left[{ }^{2}\right]$.
\end{abstract}

Together, the energy spread correction and the channel-depth conversion comprise an accurate and convenient hydrogen depth profiling method.

PACS: $\quad$ 61.18. Bn

61.43. Bn

61.72.Ss

61.72. $\mathrm{Tt}$

Key Words: depth profiling, energy spread, geometric alignment, energy calibration

Corresponding Author:

Raymond D. Verda

Mail Stop G755

Los Alamos National Laboratory

Los Alamos, NM 87545

verda@lanl.gov

5056656685

fax 5056678021 


\section{Introduction}

Interest in hydrogen depth profiling of materials has driven much of the development of elastic recoil detection analysis (ERDA) $\left[{ }^{3}\right]$. This work introduces an accurate and convenient method of hydrogen depth profiling by reflection $\left[{ }^{4}\right]$ ERDA.

In order to perform accurate depth profiling by ERDA it is important that the experimental techniques ensure accurate and reproducible geometric alignment and energy calibration. Considerations relevant to reflection ERDA--sample tilt angle, eucentric position, incident particle beam steering, and energy calibration--are treated in Verda, et al. $\left[{ }^{5}\right]$. That work introduces a laser alignment technique for the tilt angle that is accurate to $0.04^{\circ}$, as well as techniques that address the previously mentioned geometric considerations and absolute energy calibration.

A technique to convert reflection ERDA spectra to depth profiles, the channel-depth conversion, was introduced by Verda, et al $\left[^{1}\right]$. The technique converts the channel axis of ERDA spectra directly to units of depth. But the channel-depth conversion does not correct for energy spread, the unwanted broadening in the energy of ERDA spectra, which can lead to errors in depth profiling. A work in progress introduces a technique to correct for energy spread in ERDA spectra, the energy spread correction $\left[{ }^{2}\right]$. The energy spread correction and the channel-depth conversion techniques, applied to ERDA spectra in that order, comprise the depth profiling method presented here. 


\section{Experimental}

Ion beam analysis (IBA) spectra of calibration standards and hydrogen-implanted silicon samples were used in this work. The IBA spectra of the standards were used to determine the energy spread correction and the channel-depth conversion applied to the ERDA spectra of the samples. Rutherford backscattering spectrometry (RBS) and ERDA spectra of the standards and samples were collected simultaneously in the reflection geometry at nominal tilt and recoil angles of $75^{\circ}$ and $30^{\circ}$, respectively. In order to examine the effect of absorber foils on the depth profiling method, ERDA spectra of the samples were taken with mylar absorber foils of nominal thickness 12 and $18 \mu \mathrm{m}$ positioned in front of the detector. The experimental arrangement, calibration standards, calibration techniques, and other pertinent information are described elsewhere [ $\left.{ }^{1,5}\right]$.

\section{Model for the Energy Spread Correction}

An excellent discussion of the components of energy spread relevant to IBA is given by Szilagyi $\left[{ }^{6}\right]$. Following standard nomenclature, the variance of the signal of an ERDA spectrum, $\Omega^{2}$, is taken as the square of the standard deviation, $\Omega$. In determining the energy spread correction we assume that the total variance, $\Omega_{\text {TотАL }}^{2}$, measured directly from a spectrum, can be expressed as the sum of its components $\left[{ }^{7}\right]$. Then

$$
\Omega_{T O T A L}^{2}=\Omega_{E N E R G Y S P R E A D}^{2}+\Omega_{A C T U A L}^{2},
$$


expresses the total variance as the sum of the energy spread components, $\Omega_{\text {ENERGY SPREAD }}^{2}$ and the actual variance in the signal from the sample, $\Omega_{\text {ACTUAL }}^{2}$. Verda, et al. $\left[{ }^{2}\right]$ presents an experimental technique to determine energy spread, which allows the actual variance of a spectrum to be determined using Eq. (1).

Since energy spread increases with increasing depth in the material $\left[{ }^{6}\right]$, the model for the energy spread correction is a function of depth. In the analysis it is convenient to express depth in terms of the channel difference, $x$, from the surface peak to a point in the ERDA spectrum corresponding to a certain depth.

The model assumes that energy spread manifests as a broadening of the ERDA spectrum about its centroid. In the model, a function defined as the correction factor, $k$, is given by

$$
k(x) \equiv \frac{\Omega_{\text {ACTUAL }}(x)}{\Omega_{\text {TOTAL }}},
$$

where the actual standard deviation is expressed as a function of depth by way of the channel difference, $x$. The correction factor scales the channel difference axis of the adjusted spectrum about its centroid, $c$, using

$$
x_{C O R R E C T E D}=k(x-c)+c .
$$

In essence, Eq. (3) is the energy spread correction. A more detailed treatment of the energy spread correction is found elsewhere $\left[{ }^{2}\right]$. Once the energy spread correction has been applied to a 
spectrum it can be transformed into a depth profile by the channel-depth conversion $\left[{ }^{1}\right]$. As with the energy spread correction, the channel-depth conversion is expressed as a function of depth by way of the channel difference, $x$.

\section{Results and Discussion}

Fig 1(a) shows two ERDA spectra of the same silicon sample implanted with $\mathrm{H}_{2}{ }^{+}$at $40 \mathrm{keV} / \mathrm{amu}$; one spectrum was taken with a $12 \mu \mathrm{m}$ absorber, the other with an $18 \mu \mathrm{m}$ absorber. The first step in the depth profiling method is to transform the ERDA spectrum into an adjusted spectrum. This is done by subtracting the value of each channel of the spectrum from the value of the channel where the surface peak centroid appears, which expresses the spectrum in terms of the channel difference, $x$. Fig. 1(b) shows the adjusted spectrum corresponding to the ERDA spectrum taken with the $12 \mu \mathrm{m}$ absorber foil from Fig. 1(a). The surface peak is omitted in the adjusted spectrum.

Also shown in Fig. 1(b) is the result of applying the energy spread correction, Eq. (3), to the adjusted spectrum. The correction to the spectrum is greater at greater channels due to the increase in energy spread with depth in the sample. Thus, the two profiles no longer have the same centroid, that of the corrected profile being the shallower. Also shown in the figure is the correction factor, $k$, which decreases with increasing channels because of the concomitant increase in the energy spread with depth in the sample.

Fig. 2(a) compares the depth profile corresponding to the corrected spectrum in Fig. 1(b) to TRIM $\left[{ }^{8}\right]$ simulations performed with the stopping data of Konac, et al. $\left[{ }^{9}\right]$ and Ziegler, et al. $\left[{ }^{8}\right]$. 
The results suggest that the Konac, et al. stopping data for hydrogen traversing silicon may be more appropriate than the Ziegler, et al. data in the energy regime typical of ion implantation.

In the work that introduces the energy spread correction $\left[{ }^{2}\right]$, the statistics of depth profiles with and without the energy spread correction were compared to TRIM simulations. Because the energy spread correction to ERDA spectra is greater at greater channels, the corrected depth profiles became more skewed toward the surface. The calculated skewness $\left[{ }^{10}\right]$ of the profiles with the energy spread correction showed better agreement with the TRIM simulations than those without the correction. The close agreement for skewness gives us confidence that profiles with the energy spread correction are more physically accurate than those without.

Fig. 2(b) shows the application of the depth profiling method to four spectra of two silicon samples implanted with $\mathrm{H}_{2}^{+}$at 30 and $40 \mathrm{keV} / \mathrm{amu}$, respectively. Each sample had a spectrum taken with a 12 and an $18 \mu \mathrm{m}$ absorber foil. The depth profiles for the $40 \mathrm{keV} / \mathrm{amu}$ implant were obtained from the ERDA spectra in Fig. 1(a). The $30 \mathrm{keV} / \mathrm{amu}$ implant was done with half the dose as that of the $40 \mathrm{keV} / \mathrm{amu}$ implant, which is apparent from the difference in at.\% concentrations in Fig. 2(b).

The experimental depth profiles, Fig. 2(b), show that the method is independent of the absorber foil used, regardless of the energy or dose of the implant. It is shown elsewhere $\left[{ }^{2}\right]$ that the channel-depth conversion gives accurate depth information independent of the absorber foil used to acquire ERDA spectra. Thus, the agreement between depth profiles for spectra collected using 
different absorber foils, and at different energies and doses, indicates that the depth profiling technique is self-consistent.

\section{Conclusion}

The depth profiling method presented here consists of the energy spread correction, which corrects for the unwanted broadening of a spectrum caused by energy spread, followed by the channel-depth conversion, which converts a spectrum to a depth profile. The energy spread correction is easily determined from IBA and simple mathematical expressions that follow from reasonable assumptions about how energy spread manifests in ERDA spectra. The channeldepth conversion gives an accurate depth conversion independent of the absorber foil used to acquire ERDA spectra. The energy spread correction and channel-depth conversion techniques, along with accurate geometric alignment and energy calibration, comprise a method to perform accurate and convenient hydrogen depth profiling by reflection ERDA.

\section{Acknowledgements}

We gratefully acknowledge contributions from Carl Maggiore, Tobias Hoechbauer, R. L. Hervig, Caleb Evans, Mark Hollander and Chris Wetteland. This work was supported by the Department of Energy, Office of Basic Energy Sciences, Division of Materials Science.

\section{Figure Captions}

Fig. 1.(a) Two spectra of the same hydrogen-implanted silicon sample, each taken with a different absorber foil. (b) The adjusted spectrum corresponding to the ERDA spectrum taken 
with the $12 \mu \mathrm{m}$ absorber foil in Fig. 1(a), its corrected spectrum, and the correction factor $k$, Eq. (2), for this example.

Fig. 2. (a) Compares TRIM simulations with the depth profile obtained from the corrected spectrum from Fig. 2. (b) Four depth profiles of two hydrogen-implanted silicon samples. Each sample had an ERDA spectrum taken with a 12 and an $18 \mu \mathrm{m}$ absorber foil, which were used for the depth profiles. The spectra from Fig. 1(a) were used for the $40 \mathrm{keV} / \mathrm{amu}$ profiles.

\section{References}

$\left.{ }^{1}\right]$ R.D. Verda, C.J. Maggiore, J.R. Tesmer, A. Misra, T. Hoechbauer, M. Nastasi, R.W. Bower, Nucl. Instr. and Meth. B, 183, (2001), 401.

$\left[{ }^{2}\right]$ R.D. Verda, J.R. Tesmer, M. Nastasi, R.W. Bower, Accepted for Publication, Nucl. Instr. and Meth. B, Ed. Ref. P5-2365.

$\left[{ }^{3}\right]$ J. Tirira, Y. Serruys, P. Trocellier, in: Forward Recoil Spectrometry, (Plenum, New York, NY, 1996), p. ix.

$\left[{ }^{4}\right]$ J. Tirira, Y. Serruys, P. Trocellier, in: Forward Recoil Spectrometry, (Plenum, New York, NY, 1996), p. 1.

$\left[{ }^{5}\right]$ R.D. Verda, J.R. Tesmer, C.J. Maggiore, M. Nastasi, R.W. Bower, Nucl. Instr. and Meth. B, 183, (2001), 391.

$\left.{ }^{6}\right]$ E. Szilagyi, Nucl. Instr. and Meth. B, 161-163, (2000), 37.

$\left[{ }^{7}\right]$ E. Rauhala, in: Handbook of Modern Ion Beam Materials Analysis, eds. J.R. Tesmer, M. Nastasi, (Materials Research Society, Pittsburgh, PA, 1995), p.15.

$\left[{ }^{8}\right]$ J.F. Ziegler, J.B. Biersack, U. Littmark, The Stopping and Range of Ions in Solids, (Pergamon, New York, NY 1985).

[ $\left.{ }^{9}\right]$ G. Konac, S. Kalbitzer, Ch. Klatt, D. Niemann, R. Stoll, Nucl. Instr. and Meth. B, 136-138, (1998), 159.

$\left[{ }^{10}\right]$ M. Nastasi, J.W. Mayer, J.K. Hirvonen, in : Ion-Solid Interactions: Fundamentals and Applications, (Cambridge University Press, Cambridge, 1996) p. 120. 
ERDA Spectra $40 \mathrm{keV} / \mathrm{amu} \mathrm{H}$ into $\mathrm{Si}$
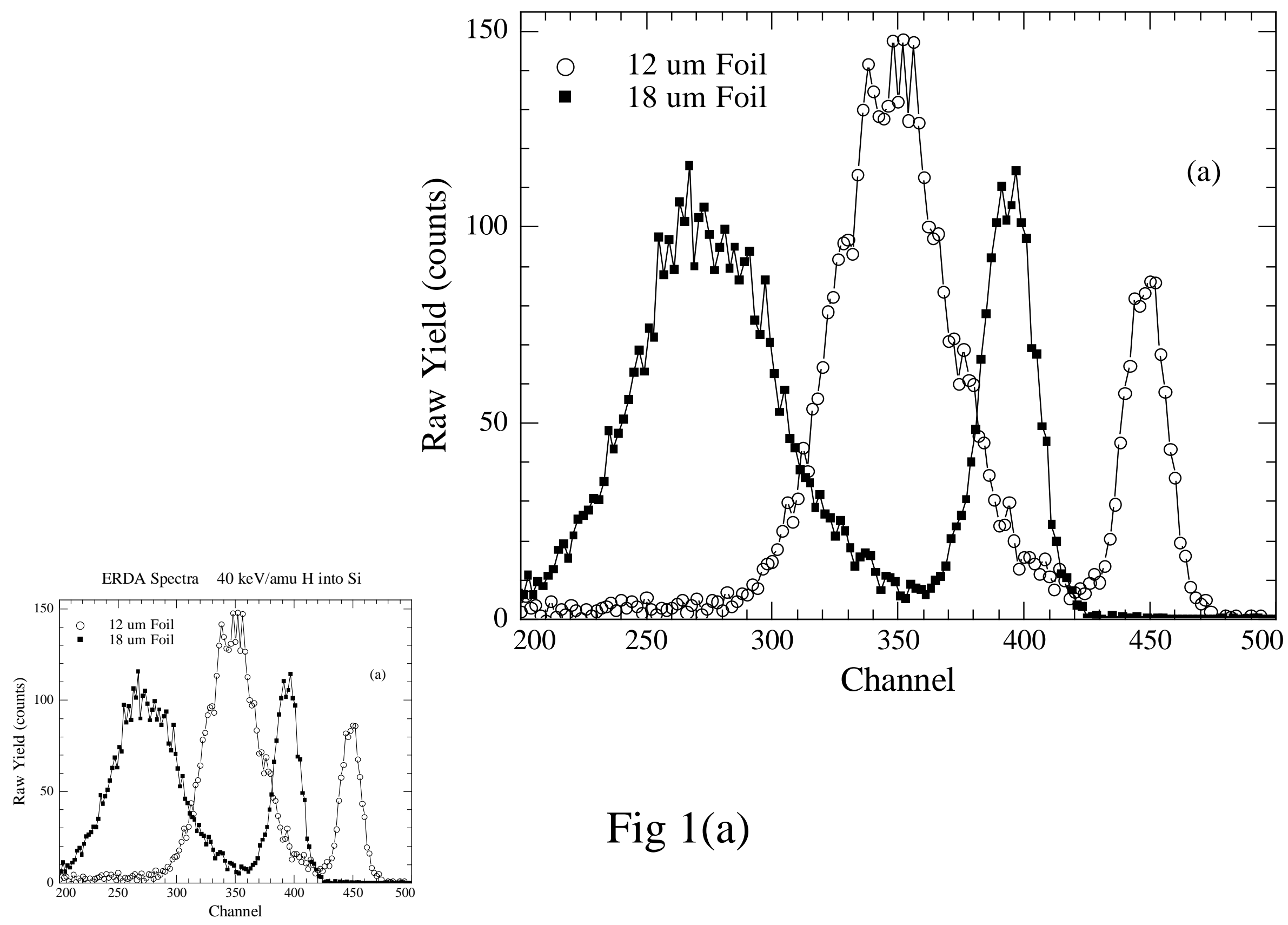

Fig 1(a) 
Adjusted and Corrected Spectra, Correction Factor

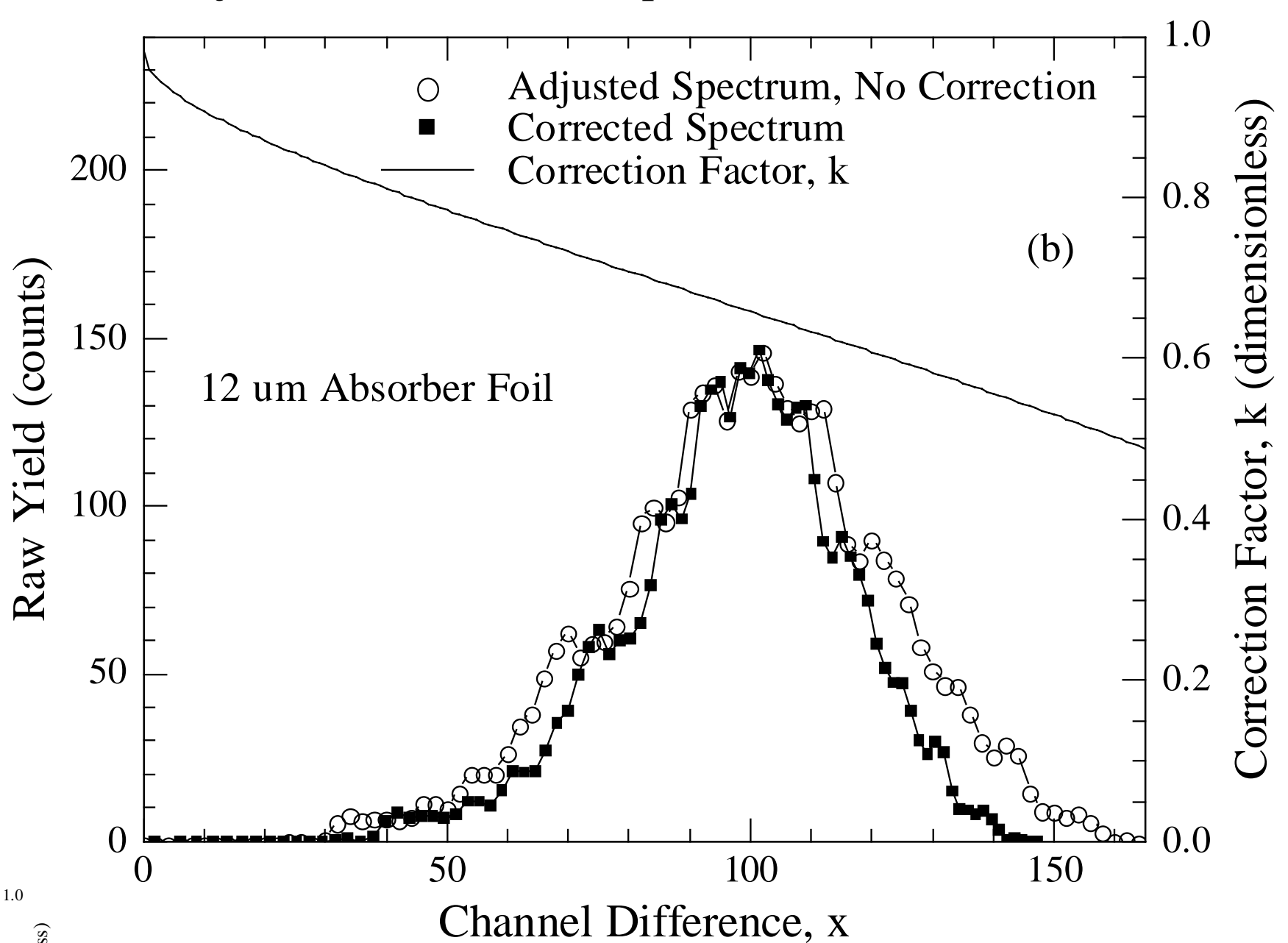

Fig 1(b)

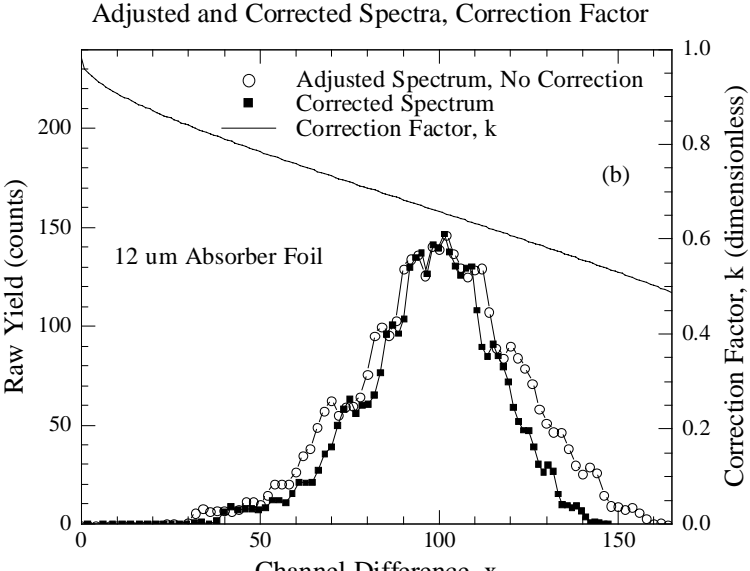


Experimental and Simulated Depth Profiles

Experimental and Simulated Depth Profiles

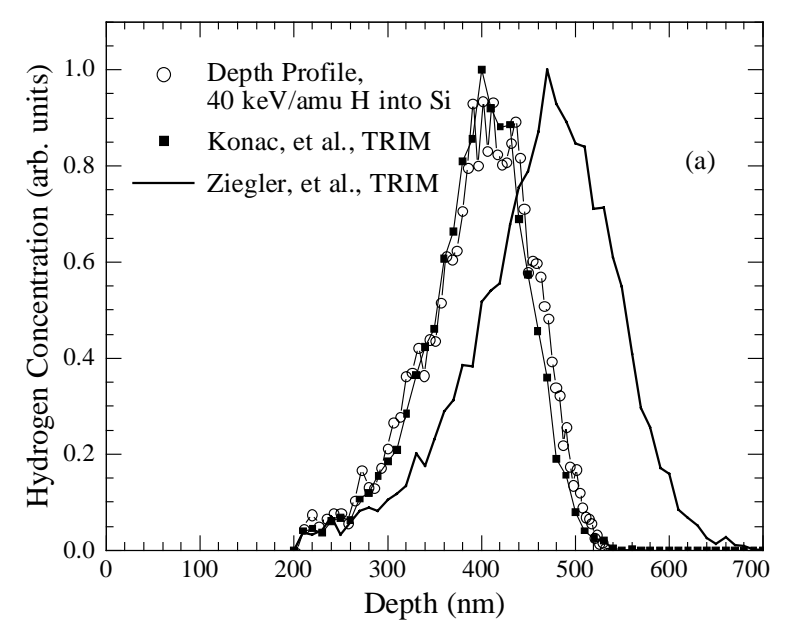

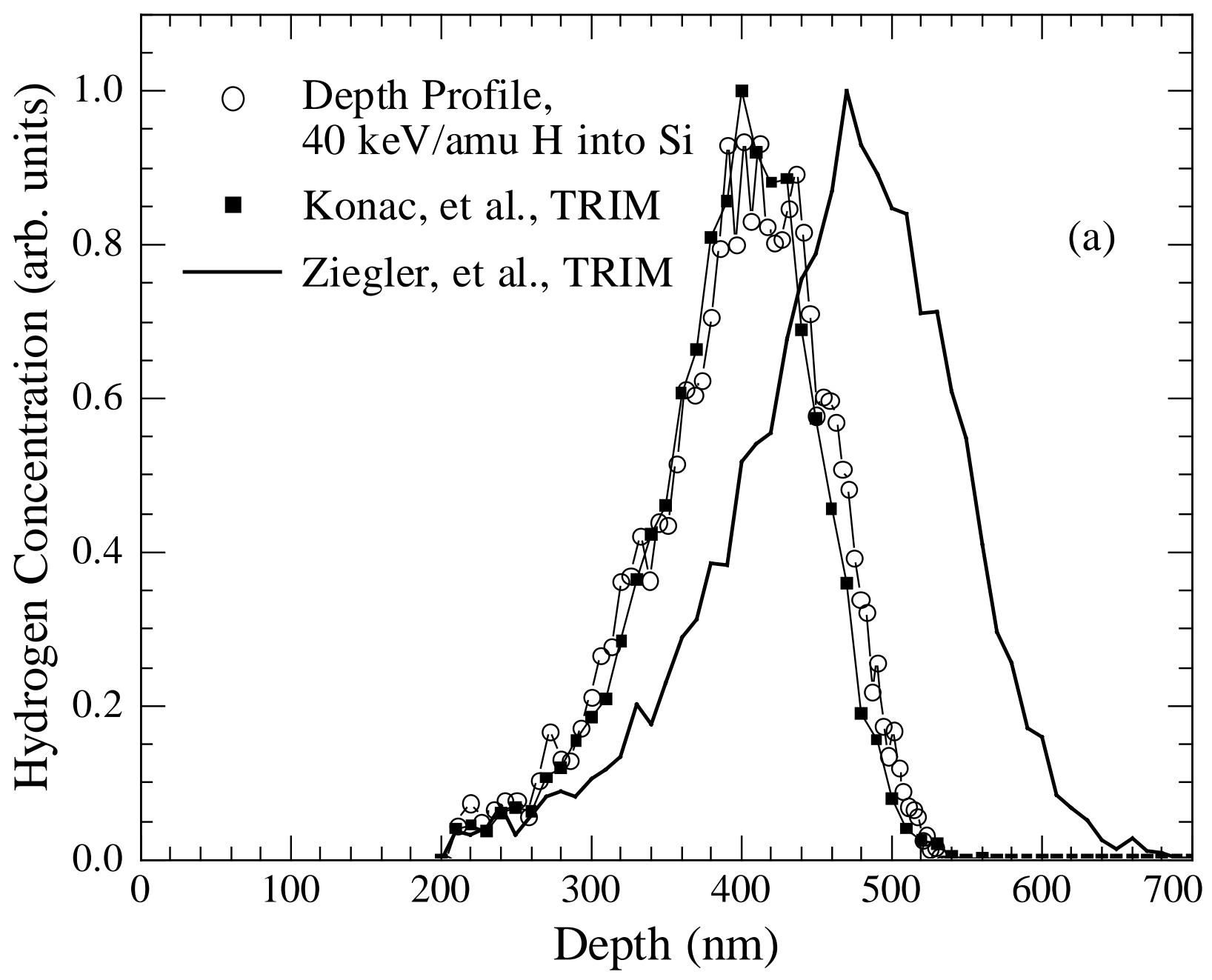

Fig 2(a) 
Experimental Depth Profiles

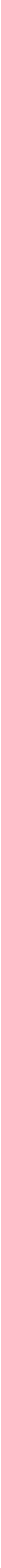

